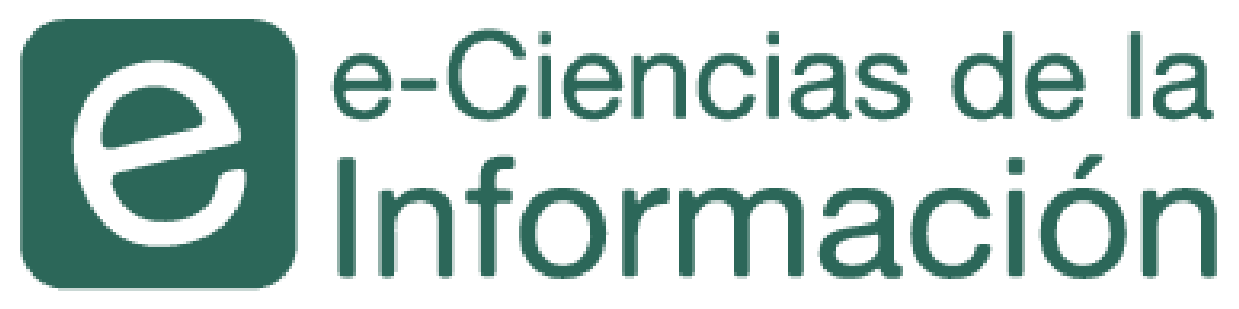

\title{
Evaluación post ocupacional en bibliotecas: una revisión sistemática
}

$>$ Celia Mireles Cárdenas $>$ Jorge Alejandro Peña Landeros Publicado 01 de julio, 2017 / Artículo científico 1

Revista electrónica semestral ISSN-1659-4142

\section{()ㅜ이우}

Escuela de Bibliotecología y Ciencias de la Información Universidad de Costa Rica

Visite el sitio web de e-Ciencias de la Información 


\section{$>$ Evaluación post ocupacional en bibliotecas: una revisión sistemática}

Post occupancy evaluation in libraries: a systematic review

$>\overline{\text { Celia Mireles Cárdenas }}{ }^{1}$ Jorge Alejandro Peña Landeros ${ }^{2}$

\section{RESUMEN}

Ante el cuestionamiento sobre cuáles son las características que los espacios bibliotecarios deben tener para ser pertinentes a las prácticas y conductas propias de los usuarios del siglo XXI, este estudio establece como objetivo general identificar las principales características de la literatura científica que refiere al tema de la evaluación post ocupacional en bibliotecas en los últimos diez años. Para lograr el objetivo establecido, se realizó una revisión sistemática de la literatura que refiere a los estudios de evaluación post ocupacional en bibliotecas en los idiomas inglés, español y portugués, publicada entre los años 2006-2016, en el Buscador de Información Global (BIG), de EBSCO, Web Of Science (WOS), Education Resources Information Center (ERIC), Red de Revistas Científicas de América Latina y el Caribe (REDALyC), DIALNET, INFOBILA, Google y Google Académico. Los resultados obtenidos muestran que existe un balance entre los estudios teóricos y empíricos sobre el tema, que son llevados a cabo, principalmente, por arquitectos y bibliotecólogos, tanto en bibliotecas universitarias como públicas, así como el empleo de diversos métodos y técnicas en su implementación. Las conclusiones enfatizan la importancia de este tipo estudios para la mejora del uso de los espacios y la optimización de los recursos invertidos en la construcción o remodelación de bibliotecas, así como del trabaio colaborativo e interdisciplinario para llevarlos a cabo.

\section{ABSTRACT}

Faced with the questioning about what are the characteristics that the library spaces must have to be relevant to the practices and behaviors of the users of the XXI century, his study establishes as a general objective to identify the main characteristics of the scientific literature that refers to the Subject of post-occupancy evaluation in libraries in the last ten years. In order to achieve the established objective, a systematic review of the literature referring to post-occupancy evaluation studies in libraries was carried out in the following languages: English, Spanish and Portuguese, published between 2006-2016, in the Academic Search Complete of EBSCO, Web of Science (WOS), Education Resources Information Center (ERIC), Network of Scientific Journals of Latin America and the Caribbean (REDALyC), DIALNET, INFOBILA, Google and Google Scholar. The results show that there is a balance between the theoretical and empirical studies on the subject that are carried out mainly by architects and librarians in both university and public libraries, as well as the use of different methods and techniques in their implementation. The conclusions emphasize the importance of this type of studies for the improvement of the use of spaces and the optimization of the resources invested in the construction or remodeling of libraries, as well as of the collaborative and interdisciplinary work to carry them out.

1 Universidad Autónoma de San Luis Potosí. Facultad de Ciencias de la Información. San Luis Potosí, MÉXICO. Orcid: orcid.org/0000-0002-7113-0705.zelia.mireles@gmail.com

2 Universidad Autónoma de San Luis Potosí. Facultad de Ciencias de la Información. Maestría en Ciencias de la Información Documental. San Luis Potosí, MÉXICO. Orcid: orcid.org/0000-0003-0229-7576.

joalpelan@gmail.com 
Palabras clave

Evaluación post ocupacional; evaluación de edificios; edificios de biblioteca; utilización del espacio de bibliotecas; arquitectura de la biblioteca.

\section{Keywords}

Post occupancy evaluation; evaluation of buildings; library buildings; library space utilization; library architecture.

\section{Introducción}

La evaluación post ocupacional se presenta como un método de evaluación viable para identificar las necesidades, percepciones y expectativas de los usuarios de bibliotecas con relación al uso de los espacios. En la actualidad, las bibliotecas tienen que responder de forma eficiente a los cambios sociales, económicos, educativos, tecnológicos e inclusive, climáticos que se presentan en las diferentes regiones del planeta. Estos aspectos están modificando los hábitos y costumbres de los usuarios con respecto a las formas de acceder y utilizar los recursos informativos.

Por su parte, los nuevos modelos basados en el aprendizaje continuo y colaborativo, así como la incorporación de las tecnologías de la información y la comunicación en los procesos formativos, entre otros factores, han generado la necesidad de proporcionar espacios físicos adecuados para responder a las nuevas realidades de los usuarios, quienes están cambiando sus hábitos, costumbres y necesidades de información conforme al desarrollo tecnológico, social y económico que el mundo enfrenta en las últimas décadas.

Cabe recordar que la situación de la economía mundial, en especial en los países latinoamericanos, ocasiona que los recursos financieros destinados a la remodelación o construcción de espacios bibliotecarios ex - proceso, sean cada vez más escasos, por lo cual la realización de investigaciones sobre las necesidades, uso, satisfacción, e impacto de los espacios físicos de las bibliotecas, permitirá, entre otras cuestiones, identificar la relación coste beneficio que demuestren que la inversión ha sido productiva, y con ello, justificar las inversiones que se realicen en este complejo rubro.

Una forma de estudiar la satisfacción y el impacto que tienen los espacios bibliotecarios en los usuarios, es a través de la evaluación en el uso de diferentes áreas, como por ejemplo, los estudios evaluación post ocupacional (POE por las siglas en inglés: Post Occupancy Evaluation), los cuales a partir de una metodología mixta, permiten obtener indicadores cualitativos y cuantitativos para integrar un análisis completo sobre el impacto y rendimiento de los espacios construidos, con relación a la opinión real de los usuarios que los ocupan (Arana, 2015).

Los estudios POE, al igual que cualquier modelo específico de evaluación, ofrecen beneficios para el mejoramiento de la calidad de los planes y programas de diseño para la edificación, remodelación, uso de los espacios y en un último alcance, pueden incidir en el establecimiento de normas y lineamientos de construcción. Sin embargo, el aspecto más importante de estos estudios es el de conseguir espacios adecuados para las personas, con base en sus propias 
opiniones, puesto que al evaluar los diversos aspectos del espacio de la biblioteca, se pueden identificar necesidades, percepciones y expectativas del usuario con relación a su uso real, con lo que además, se permite que el usuario entienda el funcionamiento de los mismos e identifique disfuncionalidades que puedan tener, con lo que se cumple el proceso de "diseño basado en las personas” al que refieren diversos proyectos sobre el tema (Gallo León, 2012).

Una característica importante de los POE, es su gran capacidad de adaptación que permite pueda aplicarse en diferentes espacios como son oficinas, escuelas, parques, o como en este caso, en bibliotecas.

POE need to be flexible to allow the various different stakeholders (design teams, clients, end users) to specify what they would like to know, and are capable of finding out [POE necesita ser flexible para permitir a los diferentes actores involucrados (equipo de diseño, clientes y usuarios) especificar qué es lo que les gusta y que es lo que se podría descubrir]. (Collins, 2014, p. 15) ${ }^{3}$

Cabe mencionar que la presente investigación, forma parte de un proyecto que lleva a cabo el Cuerpo Académico "Servicios y Políticas de Información", de la Facultad de Ciencias de la información de la Universidad Autónoma de San Luis Potosí, (UASLP) en México, en el que se pretende analizar las experiencias relacionadas con el diseño, construcción, remodelación, uso, satisfacción, e impacto de los espacios físicos de las bibliotecas, y con ello, identificar fortalezas y áreas de oportunidad para la propuesta de mejoras que contribuyan a una oferta de servicios de información pertinentes al contexto social, educativo y ambiental que prevalece en el mundo.

En México, la evaluación de los espacios bibliotecarios representa una línea de investigación poco explorada y son pocos los estudios que se han realizado para conocer cuáles son las características que tienen los edificios construidos ex profeso o no, que ocupan las bibliotecas públicas, privadas, escolares, universitarias o de investigación. En términos generales, únicamente en los diagnósticos que ha publicado el Consejo Nacional Para Asuntos Bibliotecarios de las Instituciones de Educación Superior (CONPAB-IES) desde el año 2000, se pueden encontrar algunos datos básicos sobre los edificios de las bibliotecas de las 34 Instituciones de Educación Superior que integran el Consejo. Por ejemplo, en el último diagnóstico publicado en el año de 2015, se identifica un universo de 1,030 bibliotecas, de las cuales 408 (39.7\%) cuentan con edificios construidos ex-profeso para bibliotecas (De acuerdo al Diagnóstico de las bibliotecas de instituciones de educación superior e investigación afiliadas al CONPAB - IES 1993 -2013, Consejo Nacional para Asuntos Bibliotecarios de Instituciones de Educación Superior, 2015). 


\section{2.}

\section{Antecedentes y conceptos}

Las evaluaciones post ocupacionales comienzan a realizarse en la década de los sesenta, debido a la detección de diferentes problemáticas que presentaban edificios del sector educativo, los cuales, a pesar de haber sido construidos con base en normas y lineamientos, presentaban deficiencias en cuestiones ambientales, de seguridad e higiene, o simplemente, no eran agradables para el usuario, por lo que profesionales de diversas áreas estructuraron metodologías y herramientas para la evaluación de los espacios de tal forma, que se permitiera identificar los focos problemáticos y, con los resultados, elaborar propuestas de mejora para su uso.

En 1960, el equipo The Building Performance Research Unit de la University of Strathclyde en Escocia, realizó el primer POE que se tiene registrado, al evaluar cincuenta escuelas. En este estudio se hizo hincapié en la revisión de las necesidades de los usuarios y la valoración de cada una de sus experiencias en relación con el uso del espacio. Posteriormente, se realizan otros POE en los Estados Unidos de Norteamérica (Tookaloo y Smith, 2015).

En un inicio, las áreas de aplicación fueron el ámbito educativo, así como edificios asistenciales del sector público y privado como hospitales, escuelas, guarderías, galerías de arte, entre otros. En estas primeras décadas de aplicaciones, se discutieron aspectos para el perfeccionamiento de la evaluación, hasta que, en la década de los setenta, se originó un modelo con mayor rigor y sistematicidad, centrado en la satisfacción de los usuarios, el cual aporto pautas y criterios para el diseño y construcción de nuevos proyectos (Gallo León, 2012).

En la década de los ochenta, los estudios POE logran importantes avances teóricos y empíricos, estructurándose poco a poco como una línea de investigación propia en países anglosajones como EUA, Inglaterra, Canadá y Alemania. Cabe destacar que en esta década los POE se aplicaron en países orientales como Pakistán, Arabia Saudita, Turquía y Taiwán, país en donde se aplican por primera vez en bibliotecas (Chen, 2015).

A partir de la década de los noventa y en el dos mil, los POE tienen un auge en el desarrollo de teoría, conceptos y definiciones desde diversas áreas como la psicología ambiental, el diseño, la arquitectura, la ingeniería espacial, la etnografía, la educación, la sociología, las ciencias de la salud, la bibliotecología, entre otras. Esta interrelación disciplinaria permitió un enriquecimiento en el avance teórico y el fortalecimiento de metodologías. En la actualidad, los POE se aplican en diferentes ciencias con diferentes enfoques, metodologías y técnicas, en ambientes y espacios de recreación social como plazas comerciales, restaurantes, zoológicos; espacios de recreación intelectual y cultural como escuelas, bibliotecas, librerías, galerías de arte; y espacios laborales como oficinas, centros de salud, entre otros, tanto de sectores institucionales públicos como privados.

Con relación a las definiciones, los POE no se encasillan en un solo concepto, puesto que el término ha evolucionado conforme a sus ámbitos de aplicación y con respecto a la interrelación disciplinaria desde la cual se lleva a cabo. De esta forma, se encuentran definiciones desde la perspectiva de la Psicología como la siguiente: "examination of the effectiveness for human 
users of occupied, designed environments [examen de la eficacia y la eficiencia para los usuarios humanos de los ambientes diseñados y ocupados]" (Zimring y Reizenstein, 1980, p. 429).

Por su parte, la definición de Preiser, tiene mucha importancia en la literatura que refiere al tema, porque explica claramente lo que se entiende por POE en la actualidad:

Is a diagnostic tool and system which allows facility managers to identify and evaluate critical aspects of building performance systematically. This system has been applied to identify problem areas in existing buildings, to test new building prototypes and to develop design guidance and criteria for future facilities [Es una herramienta diagnóstica y un sistema que permite a los administradores de instalaciones identificar y evaluar aspectos físicos del desempeño del edificio sistemáticamente. Este sistema bien aplicado identifica problemas en áreas existentes de edificios, probar nuevos prototipos de edificación y desarrollar directrices y criterios para futuras instalaciones]. (1995, p. 19)

Sin embargo, la siguiente definición precisa lo que son estas evaluaciones en el ámbito bibliotecario, por lo cual consideramos es la más adecuada a utilizar:

Which is the process of systematically evaluating the performance of building afther the have been built and occupied for some time, grew out of an awareness of the value of visiting libraries prior to embarking on a new building Project [Proceso sistemático de evaluación técnica del desempeño de la construcción, después de que se ha inaugurado y ocupado durante algún tiempo, en donde se crea una conciencia del valor de visitar y analizar las bibliotecas antes de emprender un nuevo proyecto de construcción]. (Latimer, 2015, p. 1)

\section{Ventajas y desventajas en la aplicación de los POE}

Existen varios despachos arquitectónicos que llevan a cabo investigaciones sobre los POE. Sin embargo, debido a la complejidad de su aplicación, son mínimas las firmas que los realizan. Entre los más importantes se encuentra el despacho Foster and Partners, el cual ha participado en la construcción de importantes edificios en ciudades como Londres, Nueva York, Madrid, Hong Kong, Pekin, Begin, Abu Dhabi, entre otros (Arana, 2015).

Lo anterior se debe a que, elaborar un estudio POE implica considerar factores multidimensionales como son el impacto que ocasionará en el cliente, en el equipo responsable de la construcción y en los usuarios. En el caso de los clientes, no siempre apoyan la realización de este tipo de estudios, ya que consideran que, al tener espacios diseñados y construidos por profesionales, éstos cuentan con los aspectos deseables, lo que ocasiona que se considere este ejercicio elevadamente costoso, en donde los beneficios no serán útiles para ellos, sino para terceros. Por otra parte, los equipos responsables del diseño, la planeación y la 
construcción, consideran que muy probablemente serán culpados por todas las deficiencias que se encuentren en relación con el espacio y lo que estas impliquen.

A su vez, los usuarios al desconocer el funcionamiento y objetivos de un espacio, así como el proceso que implica llevar a cabo un estudio POE, pueden distorsionar las respuestas respecto a su satisfacción, ya que en la mayoría de los casos exageran las condiciones negativas a manera de crítica y dejaran de lado las áreas de oportunidad o los buenos aspectos que tengan los espacios.

Además, se deben de considerar los elementos técnicos (revisión de los niveles de rendimiento en relación con los sistemas de construcción y los equipos técnicos), y los elementos funcionales que evalúan la facilidad con la que se utiliza el edificio de forma eficaz y eficiente. Por tanto, para llevar a cabo un estudio POE, es necesario en primera instancia, realizar un esfuerzo de convencimiento de las partes involucradas, ya que implica una enorme tarea de esfuerzos, un trabajo en conjunto y de apoyo económico, es por ello que la mayoría de los sectores que los aplican son instituciones privadas, mientras que en el sector público es casi inexistente este tipo de evaluaciones (Gallo León, 2012).

\section{Metodología para la selección de documentos}

Las ventajas que presentan las revisiones sistemáticas de la literatura, es la objetividad en identificar y evaluar los estudios que refieren a un tema, lo que brinda una mayor consistencia en los resultados y conclusiones que se obtengan. De esta forma, se puede concebir que: "una revisión sistemática es un estudio de revisión, sobre una pregunta concreta, en el que se utiliza una metodología científica claramente explicitada, para la identificación, selección y valoración de estudios, sintetizando sus resultados" (Meseguer, 2014, p. 303).

Para el logro del objetivo general establecido que es: describir las principales características de la literatura científica que refiere al tema de la evaluación post ocupacional en bibliotecas en los últimos diez años, se aplicó una estrategia para la búsqueda y recuperación de información, con base en la definición de descriptores en los idiomas más predominantes en la literatura científica de América Latina como lo son el español, el portugués y el inglés. Los términos se consultaron en el Tesauro de la United Nations Educational, Scientific and Cultural Organization (UNESCO, 2017) y el Tesauro Latinoamericano en Ciencia Bibliotecológica y de Información (TELACIBIN) (Naumis Peña, Iglesias Maturana, Osuna Dumont y Espinosa Ricardo, 1999).

Al realizar una una revisión previa, se encontró que la búsqueda por el término "post occupancy evaluation" o sus siglas POE, arrojaban resultados muy generales que no referían al tema. Por tanto, se identificó el término de "evaluación", como el más adecuado, y con ayuda del operador booleano AND las palabras "post-ocupacional", AND "bibliotecas", tanto en inglés, español y portugués (Tabla 1). La búsqueda con los descriptores se realizó en el campo de título y en el texto completo del documento. Se especificó la temporalidad de 2006 -2016 debido al interés de obtener experiencias recientes de estos estudios aplicados en bibliotecas, además de que se delimitó la recuperación de documentos sólo a los que cuentan con validez de arbitraje, como son libros, capítulos de libros, artículos arbitrados, actas de congresos y tesis. 
TABLA 1

Descriptores utilizados en las búsquedas en los campos de título y texto completo, en los idiomas español, inglés y portugués

\begin{tabular}{|c|c|c|}
\hline Español & Inglés & Portugués \\
\hline $\begin{array}{c}\text { Evaluación AND post } \\
\text { ocupacional AND bibliotecas }\end{array}$ & $\begin{array}{c}\text { Post occupancy AND evaluation } \\
\text { AND libraries }\end{array}$ & $\begin{array}{c}\text { avaliação AND pós ocupacional } \\
\text { AND bibliotecas }\end{array}$ \\
\hline
\end{tabular}

Fuente: Elaboración propia de los autores.

La búsqueda se realizó durante el periodo de mayo de 2016 a febrero de 2017, en el Buscador de Información Global (BIG), herramienta de EBSCO que integra todos los recursos con los que cuenta el Sistema de Bibliotecas de la UASLP. Así como en Web Of Science (WOS), Education Resources Information Center (ERIC), Red de Revistas Científicas de América Latina y el Caribe (REDALyC), DIALNET, INFOBILA, y se complementó con una búsqueda en Google Académico.

Por último, se realizó una búsqueda manual en Google libre, con la finalidad de identificar resultados que no se hubieran obtenido con la búsqueda realizada en las bases de datos establecidas.

\section{Resultados de la búsqueda y selección de documentos}

Con base en las especificaciones ya señaladas, se recuperaron 408 registros en BIG, los cuales se sometieron a tres filtros para garantizar la pertinencia en el tema de interés: En el primer filtro, se eliminaron 30 registros que hacían referencia a conferencias, simposios sin registro, repeticiones de registros, reportes, revisiones de artículos, títulos de publicaciones periódicas, discusiones, resúmenes de conferencias, registros sin datos y patentes. Sin embargo, al revisar las características de los 378 registros restantes en el título, resumen y palabras clave, se obtuvo con gran sorpresa, que solo cuatro documentos hablaron sobre el POE en bibliotecas, debido a que el sistema recupero los términos por separado que se encontraban en cualquier parte del documento, pero no significó que hablaran sobre el tema que se estaba buscando, y en otros casos, se trataba del mismo documento (documento repetido), pero recuperado de diferente fuente. En este sentido, la revisión manual de cada documento es la que apoyó la sección final los mismos.

En Web of Science se aplicó la misma estrategia de búsqueda, con lo cual se obtuvieron un total de cinco registros bibliográficos de los cuales sólo dos cumplieron los requisitos solicitados, puesto que uno refería a la evaluación de bibliotecas, pero no a los POE, otro versaba sobre evaluación de edificios en general, y el tercer registro ya se había recuperado en BIG. En Google Scholar, únicamente, se obtuvo un documento, mientras que en Google libre se obtuvieron trece referencias. Por su parte, DIALNET, REDALyC, ERIC e INFOBILA no arrojaron resultados. 
En total se recuperaron veinte documentos en texto completo con modalidades como: en formato PDF, en Full Text Finder con una liga directa desde BIG, con un link directo a la base de datos y, por último, se solicitaron por compra cuatro artículos.

En relación con el número de documentos recuperados, nos surge el cuestionamiento sobre la estrategia de búsqueda elaborada, misma que se aplicó en diferentes lapsos con resultados variables en las mismas bases de datos. Sobre todo, a propósito de los resultados obtenidos en BIG, nos llama la atención, ya que, a pesar de haber delimitado la búsqueda por tipo de biblioteca en la revisión manual, se encontró que se referían a estudios POE en casas de habitación, hospitales, escuelas, oficinas, comercios, edificios universitarios, entre otros; así como muchos de ellos no tenían que ver con las evaluaciones POE. De esta forma, la búsqueda manual refiere a la recuperación de documentos a partir de la identificación, las revisiones bibliográficas, las sugerencias y el conocimiento personal del tema, lo que arrojó mayores resultados.

Con lo cual se recuperaron un total de veinte documentos conformados, por diez artículos, cinco tesis, un libro, un capítulo de libro y tres actas de simposio (Figura 1). Con relación al número de publicaciones por año, 2015 es el que mayor número de referencias tiene con cuatro (Figura 2). Respecto al idioma se detectó un mayor número de documentos en inglés (diez), seguido del portugués (seis), tres en español y uno en italiano, el cual también se incluyó en el análisis. Por su origen, seis documentos se publicaron en Estados Unidos, seis en Brasil, dos en México y seis en diversos países del mundo (Figura 3).

Con relación al contenido de los documentos, se encontró una rica diversidad de tópicos, como se muestra en la siguiente sección.

\section{FIGURA 1}

Número de publicaciones sobre evaluación post ocupacional en bibliotecas según tipología de documento, 2006 a 2016, recuperados en BIG, Web of Science, Google

\section{Scholar y Google libre}

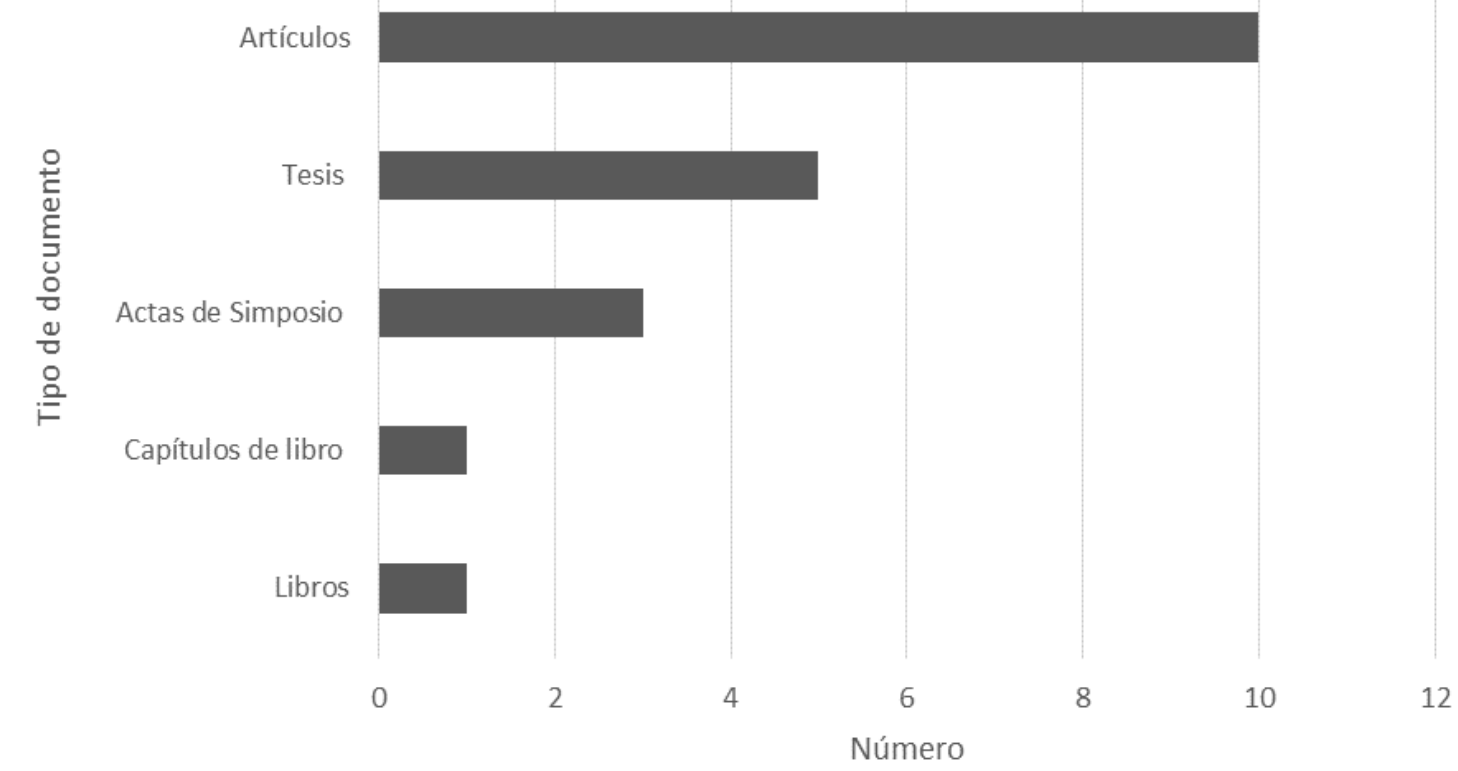

Fuente: Elaboración propia de los autores. 
FIGURA 2

Número de publicaciones sobre evaluación post ocupacional en bibliotecas según año, del 2006 a 2016, recuperado en BIG, Web of Science, Google Scholar y Google libre

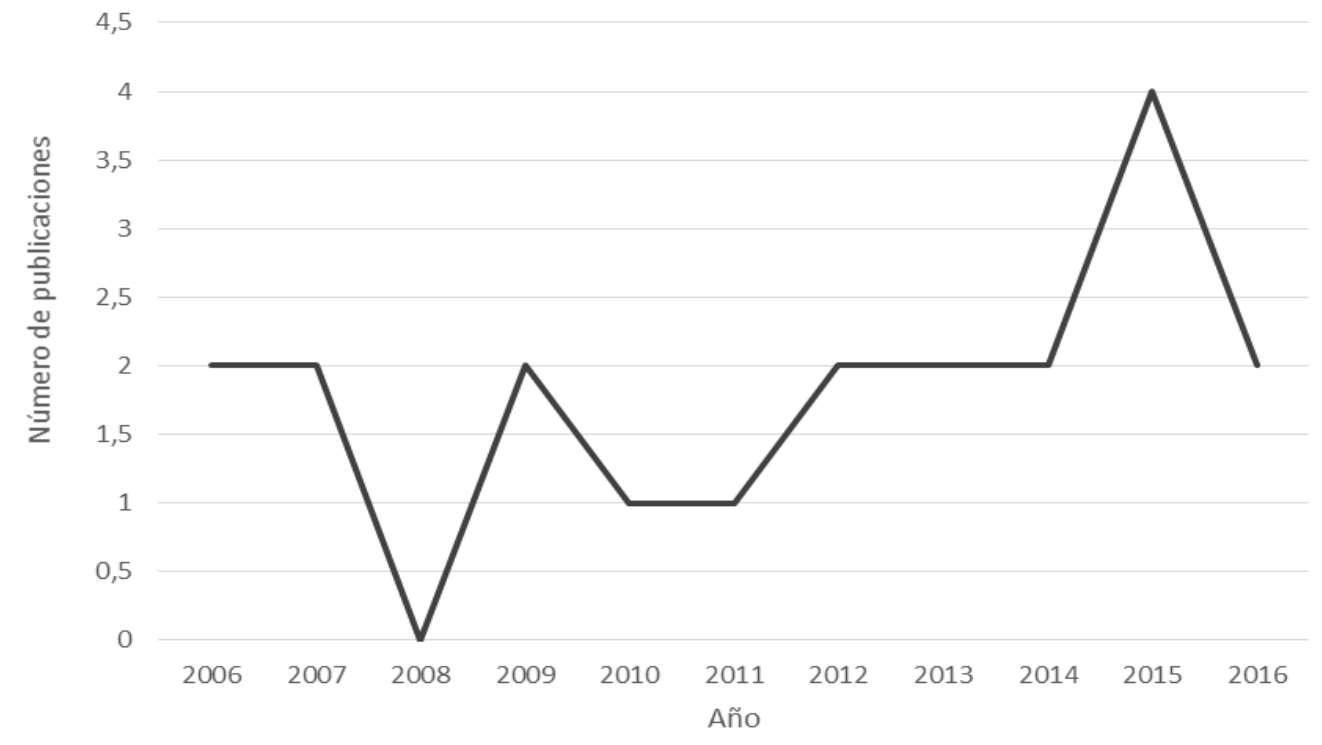

Fuente: Elaboración propia de los autores.

FIGURA 3

Número de publicaciones sobre evaluación post ocupacional en bibliotecas del 2006 a 2016 según país, recuperados en BIG, Web of Science, Google Scholar y Google libre

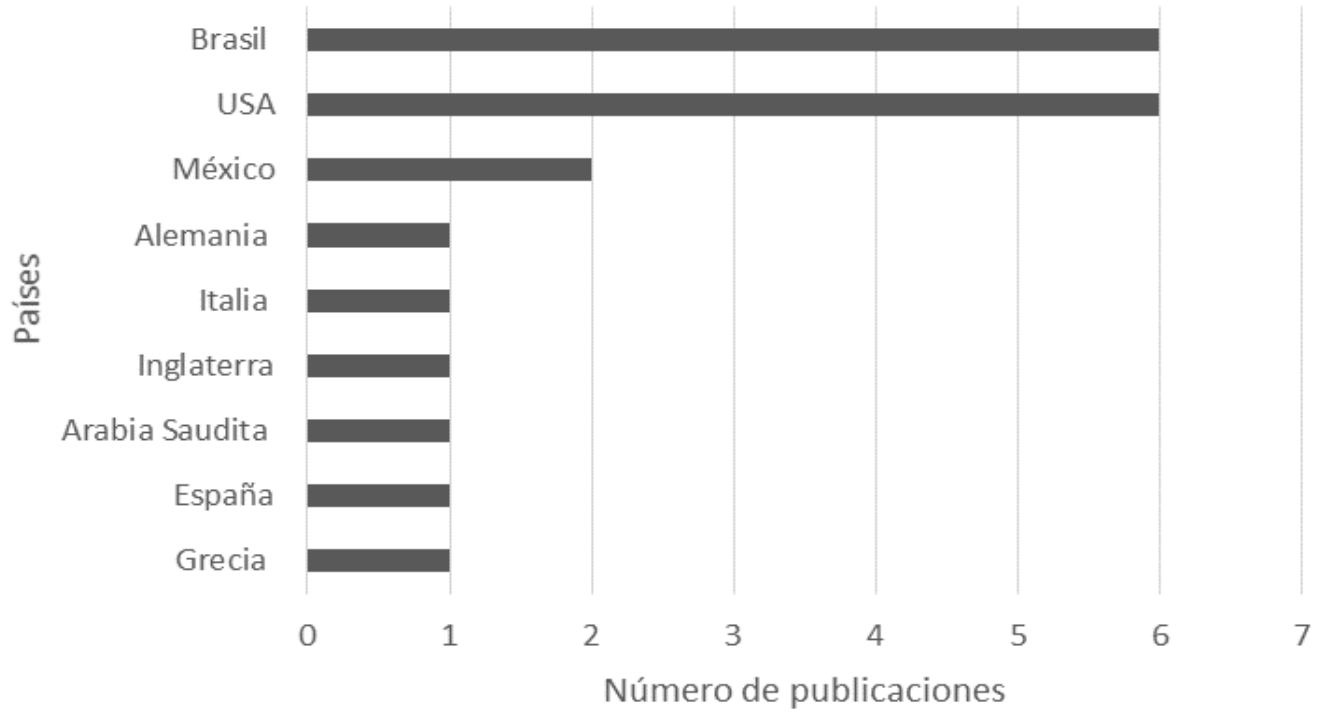

Fuente: Elaboración propia de los autores. 
La bibliografía recuperada refiere a catorce estudios sobre bibliotecas universitarias y seis en bibliotecas públicas. Desde la perspectiva teórica bibliotecológica, destaca el libro Postoccupancy evaluation of library buildings editado por la International Federation of Library Associations and Institutions (Latimer y Sommer, 2015), el cual, además de abordar la teoría especializada sobre el tema, recopila experiencias y casos de éxito en la aplicación de estudios POE a nivel internacional. Esta obra está compuesta en varias secciones y en la introducción Latimer refiere que la obra surgió como una iniciativa del equipo Library Buildings and Equipment (LBE) de IFLA.

En esta obra se encuentran aspectos teóricos, fundamentos e importancia de la evaluación de los espacios de bibliotecas a través de metodologías innovadoras, con el objetivo de aprender de la experiencia y realizar una retroalimentación en el uso de los espacios. Se exponen, además, experiencias de arquitectos que han realizado estos estudios, como Romero (2015) que describe la aplicación de estudios POE en bibliotecas públicas de Barcelona, con el objetivo de determinar si las mismas cumplían con las expectativas y funciones para las que fueron construidas, o como Chen (2015) que explica la aplicación de esta evaluación en bibliotecas de isla de Taiwan en la República China.

Además de relatar la aplicación de estas evaluaciones en bibliotecas de EUA, o en Europa, es importante resaltar que el equipo de LBE-IFLA, propone un cuestionario en idioma inglés, francés, alemán y español, para que sirva como instrumento para la elaboración de un POE en cualquier tipo de biblioteca.

En el mismo sentido de la obra anterior, Arana (2015), Gomes (2007) y Sbiroli (2013) describen desde la perspectiva arquitectónica, los antecedentes, definiciones, características, estructura, metodología y beneficios de los POE en bibliotecas. Adicionalmente, Cranz y Cha (2006), Cranz, (2013), Cha y Kim (2015), y Matetern (2007) describen experiencias en la aplicación de POE en bibliotecas públicas y resaltan la importancia del trabajo colaborativo entre arquitectos y bibliotecarios para el diseño de espacios y la opinión de los usuarios para el logro de los objetivos establecidos en las evaluaciones, aspectos en los que Gallo (2012), Mireles, Arana y Peña (2016) y Peña (2016) coinciden al reiterar la necesidad de realizar estas investigaciones en bibliotecas universitarias.

Un aspecto relevante por destacar en el análisis de los documentos recuperados, es la realización de estos estudios desde la perspectiva arquitectónica, como son los trabajos de Adhami (2011), Bernier, Males y Rickman (2014), quienes aplicaron una metodología muy estructurada en sus estudios, que les permitió realizar propuestas de mejora y algunas recomendaciones para futuras investigaciones. En el contexto arquitectónico brasileño Azevedo et al. (2015), Cristianini y Moraes (2010), Do Nascimento, Yendo, Siguemoto y Tenorio (2012), Dos Santos, Marques, Brandão y Nogueira (2009), Penha (2009), se distinguen por la combinación de varios instrumentos y herramientas durante el proceso de evaluación, los cuales se realizaron, mayormente, en bibliotecas universitarias. 
Las finalidades de los POE, así como las metodologías utilizadas son de naturaleza variada. Por ejemplo, Azevedo et al. (2015) realizaron un estudio práctico en el que utilizaron fotografías, cuestionarios, entrevistas y mapas visuales que les permitieron obtener una visión general sobre el rendimiento ambiental del edificio. Por su parte, Cristianini y Moraes (2010) elaboraron un estudio utilizando como herramientas un cuestionario con tres bloques de preguntas, cuyas respuestas fueron analizadas y presentadas con porcentajes. Do Nascimento et al. (2012) utilizaron el estudio POE para analizar el rendimiento físico del edificio y medir la satisfacción de los usuarios en la biblioteca universitaria.

A su vez, Dos Santos et al. (2009) aplicaron un estudio práctico utilizando como herramienta un cuestionario para alumnos, profesores y funcionarios sobre la infraestructura, instalaciones, cuestiones estéticas, acústica e iluminación de la biblioteca y reiteran la importancia del trabajo interdisciplinar, aspecto que Penha (2009) reitera en su trabajo. Interesante resultan los estudios de DeClercq y Cranz (2014), el cual se aplicó en una biblioteca de la University of California, Berkeley, con el objetivo investigar las conductas sedentarias de los usuarios, así como el de Bernier et al. (2014), que refiere a la evaluación de 257 bibliotecas de instituciones públicas federales en EUA, en donde participaron en su mayoría jóvenes estudiantes y en las que se analiza el espacio de la biblioteca, como un espacio cultural y democrático, además de un servicio que debe de estar en constante evaluación y mejoramiento.

Estos últimos aspectos, son abordados por Hassanai y Mudhei (2006), quienes llevaron a cabo un POE en la biblioteca de investigación de la Universidad King Fahd de Petróleos Minerales en Arabia Saudita, para determinar si las decisiones de diseño hechas por profesionales están proporcionando el rendimiento que requieren los usuarios.

Al llevar a cabo el análisis de los documentos, se detectó la mención de 18 métodos, técnicas, herramientas o instrumentos utilizados para la elaboración de los POE, como son: la investigación documental, la entrevista, el cuestionario, la encuesta, la observación, el análisis fotográfico, el tutorial o Walkthrough, el estudio comparativo, el mapa visual, la visita de campo, el mapa de comportamiento, el grupo focal, el análisis de contenido, el mapa de deseos, el poema mental, el contacto con usuarios, el análisis del discurso y el análisis de información. Cabe aclarar que los términos presentados corresponden tal cual se mencionan en los artículos.

Por último, al revisar la bibliografía se identificó que los documentos más citados por los autores son: "Post-occupancy evaluation", de la autoría de Preiser, White y Rabinowitz, (1988) y "Post-occupancy evaluation an overview" de Zimring y Reizenstein (1980), los cuales sirven como bases teóricas para hablar del POE en general. En el ámbito de las bibliotecas, se destaca que la obra más citada es Post-occupancy evaluation of library buildings (Latimer y Sommer, 2015), editada por la IFLA y una de las autoras más destacadas es Cranz Galen. 


\section{Conclusiones}

Se concluye que los POE apoyan a la comprensión de las bibliotecas desde un punto de vista técnico y funcional por parte de los usuarios, aspecto que Mattern (2007) confirma al indicar que, entre más personas estén involucradas en este tipo de procesos, más satisfechos estarán los usuarios que utilizan los espacios bibliotecarios.

Con base en las lecturas analizadas, se identifica que la realización de un POE implica considerar de preferencia que el edificio tenga no más de cinco años de construido, puesto que se encontraron experiencias de aplicación con edificios con mayor antigüedad de construcción, que exista el interés de las autoridades y equipo de construcción en participar, tener claros los antecedentes, misión, visión y objetivos de la biblioteca con el fin de contextualizar, identificar los actores y usuarios relacionados con la construcción y el uso el edificio.

En los aspectos metodológicos, aun cuando las herramientas más utilizadas son el cuestionario y la entrevista, la variedad de métodos y técnicas empleadas en los POE, dan cuenta de la flexibilidad de adaptación a distintos entornos de estas evaluaciones, lo que permite su aplicación en todo tipo de bibliotecas, puesto que los resultados además de servir de base para propuestas de mejora, también pueden ser utilizados para otras finalidades como el estudio de las conductas y satisfacción de los usuarios con relación a la infraestructura y el rendimiento ambiental del edificio.

El trabajo colaborativo e interdisciplinario se presenta como la mejor opción en la aplicación de un POE, por lo que hay que establecer condiciones para involucrar a cada una de las partes que participan en el uso de los espacios de la biblioteca como son: el equipo encargado de la construcción, las autoridades, el personal y los usuarios de la biblioteca.

Por último, se constata que los POE son una importante opción para realizar mejoras en el uso de los espacios y en la elaboración de programas de necesidades que sirvan de base para realizar futuros proyectos, remodelaciones, o construcciones, con el fin de evitar grandes costes de construcción, obsolescencia rápida de los espacios y trabajar a partir de las buenas prácticas que encaminen a la biblioteca hacia el éxito. 


\section{Referencias}

Las referencias marcadas con un asterisco indican estudios comprendidos en la revisión sistemática.

${ }^{*}$ Adhami, N. (2011). Post-occupancy evaluation of Library West's interior design: A method to explore pre-design research and programming (Tesis de maestría inédita, University of Florida). Recuperado de http://etd.fcla.edu/UF/UFE0042985/adhami n.pdf

*Arana, G. N. (2015). Los estudios pos-ocupacionales como cuestión ética: Los edificios bibliotecarios. En C. C. Mireles (coord.), Normas para la construcción de bibliotecas: una aproximación para su evaluación (pp. 135-152). San Luis Potosí, México: UASLP.

*Azevedo, G. A. N., Martorelli, C. M., Pedroso, E. S. R., Guerra, J. M., Oliveira, J. S., Mattos, P. F., Games, R. F. D., y Costa, R. (2015). Biblioteca Lúcio Costa Fau/Ufrj: Avaliação PósOcupação Como Suporte À Tomada de Decisão. Ponencia presentada en el 15to ErgoDesign: Congresso Internacional de Ergonomia e Usabilidade de Interfaces Humano-Tecnología, Universidade Federal de Pernambuco, Caruaru, Brasil. Recuperado de http://pdf.blucher.com.br.s3.amazonaws.com/designproceedings/15ergodesign/50E102.pdf

*Bernier, A., Males, M., y Rickman, C. (abril, 2014). "It Is Silly to Hide Your Most Active Patrons": Exploring User Participation of Library Space Designs for Young Adults in the United States. The Library Quarterly: Information, Community, Policy, 84(2), 165-182. doi:10.1086/675330

${ }^{*}$ Cha, S. H., y Kim, T. W. (2015). What Matters for Students' Use of Physical Library Space? The Journal of Academic Librarianship, 41(3), 274-279. doi:10.1016/j.acalib.2015.03.014

Chen, K. (2015). A Review of post-occupancy evaluation of libraries in Taiwan from the perspective of the architecture profession. En K. Latimer y D. Sommer (Eds.), Postoccupancy evaluation of library buildings (IFLA Publications Series 169, pp. 98-104). Alemania: IFLA.

${ }^{*}$ Cranz, G. (2013). How post-occupancy evaluation research affected design and policy at the San Francisco Public Library. Journal of architectural and planning research, 30(1), 7790. Recuperado de https://www.jstor.org/stable/43030994?seq=1\#page scan tab contents 
${ }^{*}$ Cranz, G., y Cha, E. (2006). Body-conscious design in a teen space: Post-occupancy evaluation of an innovative public library. Public libraries, 45(6), 48-56. Recuperado de http://www.ala.org/pla/sites/ala.org.pla/files/content/publications/publiclibraries/pastissues Inovdec2006pl.pdf

${ }^{*}$ Cristianini, G. M. S., y Moraes, J. S. (2010). Construção De Bibliotecas: considerações e avaliação pós-ocupação. En Impacto das Tecnologias de Informação na Gestão da Biblioteca Universitária. Simposio llevado a cabo en XV Seminario Nacional de Bibliotecas Universitarias, Sao Paulo, Brasil.

Collins, E. (2014) Architects and research-based knowledge: a literature review. Londres: RIBA.

Consejo Nacional para Asuntos Bibliotecarios de Instituciones de Educación Superior. (2015). Diagnóstico de las bibliotecas de instituciones de educación superior e investigación afiliadas al CONPAB-IES 1993-2013. La Paz, Baja California Sur, México: CNABES y UABCS. Recuperado de

http://www.conpab.org.mx/Documentos/Publicaciones/DiagnosticoBibliotecas\%20CONP AB 2015.pdf

*DeClercq, C., y Cranz, G. (2014). Moving Beyond Seating-centered Learning Environments: Opportunities and Challenges Identified in a POE of a Campus Library. Journal Of Academic Librarianship, 40(6), 574-584. doi:10.1016/j.acalib.2014.08.005

*Do Nascimento S. G., Yendo, J., Siguemoto, S., y Tenorio, S. (2012). Avaliação PósOcupação em Bibliotecas Universitárias: o Caso da Biblioteca da Unesp/Campus Bauru. En A Biblioteca Universitária Como Laboratório na Sociedade na Informação. Simposio llevado a cabo en XVII Seminario Nacional de Bibliotecas Universitarias. Sao Paulo, Brasil. Recuperado de http://www.snbu2012.com.br/anais/pdf/4R6H.pdf

${ }^{*}$ Dos Santos, E. R. M., Marques, M. C., Brandão, L. C., y Nogueira, G. (noviembre, 2009). Avaliação do espaço construído: uma análise da biblioteca universitária do UniCEUMA. En IX Simpósio Brasileiro de Qualidade do Projeto no Ambiente Construído. Brasil: Universidade e Sao Paulo. Recuperado de http://www.iau.usp.br/ocs/index.php/SBQP2009/SBQP2009/paper/view/148

*Gallo León, J. P. (2012). Forma y función de los edificios de bibliotecas universitarias: herramientas para su evaluación (Tesis doctoral inédita, Universidad de Murcia). Recuperado de https://digitum.um.es/xmlui/bitstream/10201/27221/1/TJPGL.pdf 
*Gomes, S. H. T. (2007). Edifícios para bibliotecas universitárias: perspectivas e diretrizes a partir da avaliação pós-ocupação (tesis doctoral inédita). Universidade de São Paulo, Brasil. doi:10.11606/T.16.2007.tde-29042010-105703

*Hassanain, M. A., y Mudhei, A. A. (2006). Post-occupancy evaluation of academic and research library facilities. Structural Survey, 24(3), 230-239. doi:10.1108/02630800610678878

Latimer, K. (2015). Introduction. En K. Latimer y D. Sommer (Eds.), Post-occupancy evaluation of library buildings (IFLA Publications Series 169, pp. 2-6). Alemania: IFLA.

Latimer, K., y Sommer, D. (Eds.). (2015). Post-occupancy evaluation of library buildings (IFLA Publications Series 169). Alemania: IFLA.

*Mattern, S. C. (2007). The new downtown library: Designing with communities. The Library Quarterly, 78(3), 340-343.

Meseguer, G. (2014). Lectura Crítica de un Metaanálisis y de una revisión sistemática. Recuperado de https://www.murciasalud.es/recursos/ficheros/136630-capitulo 11.pdf

*Mireles Cárdenas, C., Arana López, G. N., y Peña Landeros J. A. (2016). Building Spaces for Citizen Participation and Collaboration: Library and Information Services. International Journal of Library and Information Science, 5(1), 1-7. Recuperado de http://www.qqml.net/papers/March 2016 Issue/514QQML Journal 2016 MirelesCarden asetal 31-37.pdf

Naumis Peña, C., Iglesias Maturana, M. T., Osuna Dumont, A. C., y Espinosa Ricardo, L. (1999). Tesauro Latinoamericano en Ciencia Bibliotecológica y de Información. México: UNAM, CUIB.

*Peña, J. A. (2016). Propuesta de un estudio de evaluación pos - ocupacional al edificio del Centro de Información Dr. Jerzy Rzedowski Rotter del Instituto de Investigación en Zonas Desérticas de la Universidad Autónoma de San Luis Potosí (tesis de licenciatura inédita). Universidad Autónoma de San Luis Potosí, Facultad de Ciencias de la Información, San Luis Potosí, México.

*Penha, L. M. D. O. (2009). Avaliação pós-ocupação de duas edificações de bibliotecas de instituição de educação superior: Centro Universitário de Brasília (UniCEUB) e Universidade Católica de Brasília (UCB) (tesis de maestria inédita). Universidade de Brasília, Faculdade de Arquitetura e Urbanismo (FAU), Brasil. 
Preiser, W. F. E. (1995). Post-occupancy evaluation: how to make buildings work better. Facilities, 13(11), 19-28. doi:10.1108/02632779510097787

Preiser, W. F. E., White, E., y Rabinowitz, H. (1988). Post-Occupancy Evaluation. Nueva York: Routledge.

Romero, S. (2015) Post-Occupancy evaluation of teen public libraries in Barcelona province. When architectural advice ends up being of real value. En K. Latimer y D. Sommer (Eds.), Post-occupancy evaluation of library buildings (IFLA Publications Series 169, pp. 98-104). Alemania: IFLA.

*Sbiroli, M. C. (2013). Valutazione dello spazio architettonico delle biblioteche attraverso la Post-Occupancy Evaluation. AIB Studi, 53(2), 17-28. doi:10.2426/aibstudi-8983

Tookaloo, A., y Smith, R. (2015). Post Occupancy Evaluation in Higher Education. Procedia Engineering, 118, 515-521. doi:10.1016/j.proeng.2015.08.470

United Nations Educational, Scientific and Cultural Organization. (2017). UNESCO Thesaurus. Recuperado de http://vocabularies.unesco.org/browser/thesaurus/en/

Zimring, C. M., y Reizenstein, J. E. (1980). Post-occupancy evaluation an overview. Environment and Behavior, 12(4), 429-450. Recuperado de http://journals.sagepub.com/doi/pdf/10.1177/0013916580124002 
¿Desea publicar su trabajo? Ingrese aquí

O escríbanos a la siguiente dirección: revista.ebci@ucr.ac.cr

(우(1)(2)

\section{1}

Origen: respuesta a una necesidad

En el año 2011, la Escuela de

Bibliotecología y Ciencias de la Información (EBCl) de la Universidad de Costa Rica (UCR) reconoció la importancia de crear nuevas y mejores alternativas para difusión de la investigación. e-Ciencias de la Información es la respuesta a un contexto actual marcado por una mayor apertura, flexibilidad, y rigurosidad en la verificación de los datos y su procesamiento.

\section{3}

Revista de la UCR

e-Ciencias de la Información es una revista científica que aborda las nuevas temáticas de desarrollo e investigación en las Ciencias de la Información, en el ámbito nacional e internacional. Así, colabora significativamente en el progreso de esta disciplina. Por sus parámetros de calidad, pertenece al grupo de las revistas más importantes de la UCR y se encuentra ampliamente indizada en los importantes catálogos.

\section{En la actualidad}

\section{Posicionamiento internacional}

Se encuentra en el Cuartil A del UCR Índex para el 2017, posicionándola como una de las mejores revistas de la Universidad de

Costa Rica, un reflejo claro y conciso sobre su calidad y trascendencia en el área apoyado por otros hitos como su ingreso a Scielo, DOAJ, Latindex y otros.
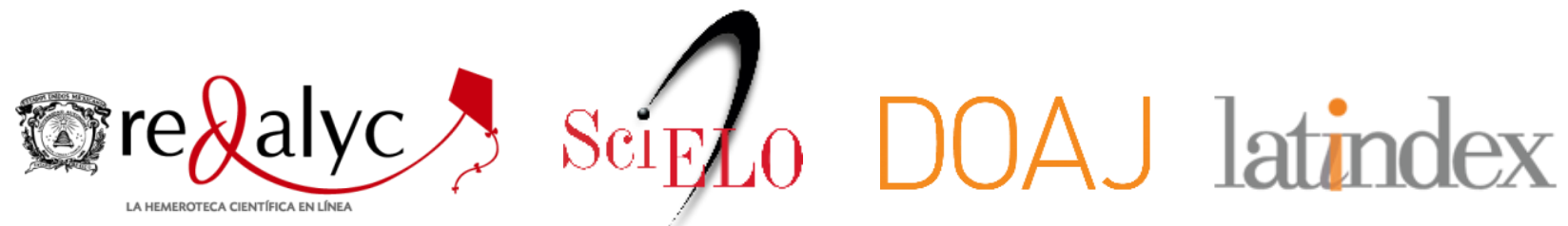

e-Ciencias de la Información está indexada en los catálogos más prestigiosos.

Para conocer la lista completa de índices, ingrese aquí 